\title{
Plank's Constant: Evaluation of Measurement Uncertainty
}

\author{
Boris Menin ${ }^{1^{*}}$ \\ ${ }^{1}$ Mechanical \& Refrigeration Consultation Expert, Beer-Sheba, Israel \\ Email: meninbm@gmail.com
}

\begin{abstract}
In this article, we present a new metric called the comparative uncertainty, according to which the least achievable relative uncertainty is calculated when measuring the Planck constant. To calculate the comparative uncertainty, information theory is used. The optimizing criterion is the number of quantities considered in the model. Its calculation is possible due to the fact that any model contains a certain amount of information about the object under study. Comparative uncertainty can be verified by field trials or computer simulations within a specified range of changes of the Planck constant. The concept of introduced uncertainty is universal and can be recommended for estimating the accuracy of measurements in the study of physical phenomena and technological processes. Examples of application of the proposed approach are discussed.
\end{abstract}

Keywords: Experiment, mathematical modelling, measurements, Planck constant, uncertainty.

\section{Introduction}

This Since Newton's law of gravity in all the basic equations of the physical-mathematical theories, together with the quantities there are appeared isolated values of physical quantities called the fundamental physical constants. In Newton's theory it is a gravitational constant $\boldsymbol{G}$, in special relativity it is the speed of light in vacuum $\boldsymbol{c}$, in general relativity they are $\boldsymbol{c}$ and $\boldsymbol{G}$, in quantum (non-relativistic) mechanics it is the Planck constant $\boldsymbol{h}$, in quantum electrodynamics they are $\boldsymbol{c}$ and $\boldsymbol{h}$.

The desire to reduce the value of uncertainty in the measurement of fundamental physical constants is due to several reasons. Firstly, achieving an accurate quantitative description of the physical universe depends on the numerical values of the constants that appear in the theories. Secondly, the overall consistency and validity of the basic theories of physics may be proved by careful examination of the numerical values of these constants as determined from different experiments in different fields of physics.

One of the seminal works close to the problem of the interpretation of measurement accuracy, as well as methods to improve the uncertainty assessment in the measurements of fundamental constants, is [1]. The authors noted that precise estimates of the fundamental constants of physics are subject to uncertainty from various sources. Reliable estimates of uncertainty are required (a) to compare the accuracy of different measurements of the same variable, (b) to evaluate the accuracy of other variables derived from them, (c) to help in defining and revising models, and most importantly, (d) to assess compliance with the physical theory with the current best measurements. In order to prove their conclusions, the authors used the Birge ratio [2], which assesses the compatibility of a set of measurements by comparing the variability among experiments to the reported uncertainties of the velocity of light, the fine structure constant and the gravitational constant.

Two quite different kinds of the fundamental physical constant uncertainty must be considered: first, the relevant variables and the functional relationships among them which we know, although the values of the key coefficients are not known; the second, when the developer is not sure of all the relevant variables, or the functional relationships among them. Often uncertainty about the form of the model is more important than uncertainty about the values of the coefficients [3].

Developers often have difficulty evaluating or even estimating the model's discrepancy from a real fundamental physical constant under realistic conditions. A lot of the model structures do not quantify the uncertainty resulting from factors such as developer knowledge, intuition, experience and environmental properties. In addition, without at least some quantification, qualitative descriptions of uncertainty convey little useful information. 
Actually, the very act of the fundamental physical constant measurement already implies the existence of the formulated physical-mathematical model describing the phenomenon under investigation. At the same time, most researchers have focused on data analysis and a calculation of the fundamental physical constant uncertainty value after formulating the mathematical model. But the unavoidable uncertainty existing before the beginning of the experiment or computer simulation, and caused only by the finite number of quantities recorded in the mathematical model of the fundamental physical constant, is generally ignored. Of course, in addition to this uncertainty, the overall uncertainty of the Planck constant measurement includes the posterior uncertainties related to the internal structure of the model, its subsequent computerization and the testing equipment characteristics: inaccurate input data, inaccurate physical assumptions, the limited accuracy of the solution of integral-differential equations, etc. Detailed definitions of many different sources of uncertainty are given in [4].

This paper represents our attempt to apply the universal metric for calculating the absolute and relative uncertainties during measurement of Planck's constant through the use of the information and similarity theories. These can also be used for other fundamental physical constants and will greatly shorten the duration of the studies and the design stage, thereby reducing the cost of the project.

\section{Preliminaries}

Different methods are used to compare the results obtained for the Planck constant measurement [5]. In the theory of measurements, it is assumed that for each dimensional measured value $\boldsymbol{U}$, there is the dimensional "presumed uncertainty" $\boldsymbol{\Delta} \boldsymbol{U}$. The full result can be represented as $\boldsymbol{U} \pm \boldsymbol{\Delta} \boldsymbol{U}$. This means that the "true value" probably lies between the maximum value $\boldsymbol{U}+\boldsymbol{\Delta} \boldsymbol{U}$ and the minimum value of $\boldsymbol{U}$ $-\Delta U$

The term "relative uncertainty" $\mathbf{r}$ is widely used in measurements of the Planck constant:

$$
\mathbf{r}=\Delta U / U
$$

Selecting the relative uncertainty is explained by the fact that the absolute uncertainty does not always give an idea of how the uncertainty is important. Further, the relative uncertainty is useful for comparing the accuracy of different measurements. It also makes the calculations of the scatter of uncertainty much easier. In addition to the above types of uncertainties, in order to weight the approached uncertainty, the conventional value of the Planck constant is given by applying the conventional (i.e., adopted) values of the Josephson constant $\mathrm{K}_{\mathrm{J}-90}$ and von Klitzing constant $\mathrm{R}_{\mathrm{K}-90}[6,7]$. Thus, the international standard of the Planck constant value was chosen to improve the uniformity of the comparison of subsequent measurements.

However, these methods for identifying the measurement accuracy do not indicate the direction in which you can find the true value of Planck's constant. At the same time, evaluation of uncertainty due to possible systematic uncertainties in the physical measurement necessarily involves an element of subjective judgment. Examination of the historical measurements and recommended values of the fundamental physical constants shows that the reported uncertainty has a consistent bias towards the underestimation of the actual uncertainty. These data are consistent with the results of persistent selfconfidence in psychological research to assess the subjective probability distributions. Awareness of these biases can help in the interpretation of the measurement accuracy, as well as providing a basis for improving the evaluation of measurement uncertainty[8].

So far, the experimental results have been inadequate. It is assumed that these discrepancies may be caused by unknown systematic uncertainties, which should be reduced to a satisfactory level. Therefore, for existing methods there should be additional investments both in improving the test benches, and in improving the measurement results, as well as in searching for a universal metric that allows to check the true-target value of the Planck constant with a given achievable relative uncertainty.

\section{Applied Techniques}

In physics, quantities are called homogeneous if they have the same dimension. Thus, the concept of homogeneity is associated with dimensions, which, in turn, requires recourse to the fundamental concept of the system of base quantities (SBQ). Among the many used in science and engineering SBQ, CGS 
(centimeter-gram-second system of units) and SI (International System of Units) are universally applicable in all fields of physics [9].

In the Giorgi system suggested in the early 20th century, on the basis of which SI was subsequently formed, seven basic quantities of measurement $(\xi=7)$ were chosen: meter, the length $L$; kilogram, the mass $\boldsymbol{M}$; second, the time $\boldsymbol{T}$; Kelvin, the thermodynamic temperature $\boldsymbol{\Theta}$, ampere, the electrical current $\boldsymbol{I}$; mole, the amount of substance $\boldsymbol{F}$; candela, the luminous intensity $\boldsymbol{J}[10]$.

The total number of quantities in the SBQ is large, but finite and can be calculated (see below). However, the dimensions attributed to physical quantities are rather conventional and contain a large fraction of arbitrariness. The only reasonable alternative is the idea of the dimensionless measurement system designed to solve the problem of dimensions[9]. In this work, the A-system was introduced: the absolute dimensionless system of physical quantities measurement based on the mathematical expressions for the initial physical constants. The author argued that the developed theory provides the tools necessary to determine any known physical constant and for "ascribing with limited or absolute accuracy the true value to any physical quantity". A significant part of the monograph is occupied by the development of mathematical formulas, linking various constants. However, it should be noted that the numerical coincidence, of course, should not be ignored, but a natural number is only entitled to be considered as truly meaningful when it is directly related to the well-known mathematical constant, and when its appearance is due to the action of universal principles. Despite the many positive aspects of the monograph, the generalization of the mathematical theory is at odds with the established approaches of terminology in the field of education of algebraic equations and related structures[11].

In [12], using basic physical arguments and the dimensional and physical analysis, the authors derived the characteristic masses and sizes of important objects in the universe in terms of just a few fundamental constants. They illustrated the unifying power of physics and the profound connections between the small and the large in the observed cosmos. The authors showed that the minimum and maximum masses of normal stars, the corresponding quantities for neutron stars, the maximum mass of a rocky planet, the maximum mass of a white dwarf, and the mass of a typical galaxy, can be expressed in terms of either the Planck mass or the Chandrasekhar mass, in combination with a certain small number of dimensionless variables. Although the authors were less interested in precision than illumination and focus on the orders of magnitude, this work[12] is interested in the fundamental connections between the small and the large in this universe we jointly inhabit.

The principle of building the dimensionless SBU based on SI with seven base quantities was suggested in [13]. The idea is to use the similarity and information theories in order to calculate the maximum possible number of dimensionless criteria in SI, $\boldsymbol{\mu}_{\mathrm{SI}}$, and a ratio $\boldsymbol{\varepsilon}$ called a comparative uncertainty. It was proved that

$$
\varepsilon=\Delta_{p m m} / S=\left(z^{\prime}-\beta^{\prime}\right) / \mu_{S I}+\left(z^{\prime \prime}-\beta^{\prime \prime}\right) /\left(z^{\prime}-\beta^{\prime}\right)
$$

All detailed definitions, and the required explanations of the quantities shown in Equation (2), are introduced below.

\subsection{Comparative Uncertainty}

3.1.1 We specify the concept of the "comparative uncertainty". Taking into account the fact that the debates and discussions on the replacement of the term "error" with the term "uncertainty" are continuing in nature and not mandatory [1, 14], we use the term "uncertainty". We understand the "uncertainty" as a discrepancy between the tested material object and the physical-mathematical model, including inaccuracy in the basic data that are used to compare with the prediction.

3.1.2 We shall henceforth use the term "comparative uncertainty" $\boldsymbol{\varepsilon}$, which is the ratio between the dimensional absolute uncertainty $\boldsymbol{\Delta} \boldsymbol{U}$ in determining the dimensional quantity $\boldsymbol{U}$ and the dimensional considered range of changes $\boldsymbol{S}^{*}$ of $\boldsymbol{U}$ proposed by Brillouin [15]:

$$
\varepsilon=\Delta U / S^{*}
$$

Absolute and relative uncertainties are familiar to physicists. As for the comparative uncertainty, it is rarely mentioned. Nevertheless, the comparative uncertainty is of great importance for the application of information theory in physics and engineering [15].

3.1.3 If $S^{*}$ is not declared, the information obtained in the measurement is impossible to define. This full a priori range of changes depends on the previous knowledge which the developer had before the 
research. If nothing is known about the studied system, then $\boldsymbol{S}^{*}$ is defined by the limits of the measuring devices used. For this reason, it would be useful to express the closeness of the model to the studied object by the comparative uncertainty.

3.1.4 We take into account that the comparative uncertainties of the dimensionless researched quantity $\boldsymbol{u}$ and the dimensional researched quantity $\boldsymbol{U}$ are equaled

$$
\left(\Delta U / S^{*}\right)=\left(\Delta U / r^{*}\right) /\left(S^{*} / r^{*}\right)=(\Delta u / S)
$$

where $\boldsymbol{S}=\boldsymbol{S}^{*} / \mathrm{r}^{*}$ is the dimensionless considered range of changes of the dimensionless measured quantity $\boldsymbol{u}, \mathrm{r}^{*}$ is the dimensional scale parameter with the same dimension that $\boldsymbol{U}$ and $\boldsymbol{S}^{*}$ have, and $\boldsymbol{\Delta} \boldsymbol{u}=\boldsymbol{\Delta} \boldsymbol{U} / \mathrm{r}^{*}$ is the dimensionless absolute uncertainty of the dimensionless quantity $\boldsymbol{u}$.

\subsection{Number of Dimensionless Criteria $\mu_{\mathrm{SI}}$}

3.2.1 The dimension of any derived quantity $\boldsymbol{q}$ can only express a unique combination of the dimensions of the base quantities in different degrees [16]:

$$
q \supset L^{l} \cdot M^{m} \cdot T^{t} \cdot I^{i} \cdot \Theta^{\Theta} \cdot J^{j} \cdot F^{f}
$$

3.2.2 $l, m \ldots f$ are the exponents of the base quantities, and the range of each has a maximum and minimum value. According to [10], the exponents of the base quantities change in the following ranges:

$$
\begin{aligned}
& 3 \leq l \leq+3,-1 \leq m \leq+1,-4 \leq t \leq+4,-2 \leq i \leq+2 \\
& -4 \leq \Theta \leq+4,-1 \leq j \leq+1,-1 \leq f \leq+1
\end{aligned}
$$

3.2.3 The exponents of the base quantities take only integer values [10], so the number of choices of dimensions for each base quantity $\boldsymbol{e}_{l}, \ldots, \boldsymbol{e}_{f}$, according to (6), is the following:

$$
e_{l}=7 ; e_{m}=3 ; e_{t}=9 ; e_{i}=5 ; e_{\theta}=9 ; e_{j}=3 ; e_{f}=3
$$

3.2.4 The total number of dimension options of physical quantities $\check{\boldsymbol{D}}$ equals $\check{D}=\prod_{l}^{f} e_{i}-1$

$$
\check{D}=e_{l} \cdot e_{m} \cdot e_{t} \cdot e_{i} \cdot e_{\theta} \cdot e_{j} \cdot e_{f}-1=7 \cdot 3 \cdot 9 \cdot 5 \cdot 9 \cdot 3 \cdot 3-1=76,544
$$

where "-1" corresponds to the occasion when all the exponents of the base quantities in formula (5) are treated to zero.

3.2.5 The value $\check{D}$ includes both required and reverse quantities, for example, the length $\mathrm{L}^{1}$ and the running length $\mathrm{L}^{-1}$. So the number of options of dimensions may be reduced at times 2 because of the fact that the researched object can be judged, knowing only one of its symmetrical parts, while others structurally duplicating this part may be regarded as empty information. This means that the total number of dimension options of physical quantities without reverse quantities equals $D=\check{D} / 2=$ 38,272 .

3.2.6 According to the $\boldsymbol{\pi}$-theorem [17], the number $\boldsymbol{\mu}_{\mathrm{SI}}$ of the dimensionless possible criteria with $\boldsymbol{\xi}=7$ the base quantities for SI will be

$$
\mu_{S I}=D-\xi=38,272-7=38,265
$$

3.2.7 $\mu_{\mathrm{SI}}$ is called the group order and corresponds to the maximum amount of information contained in SI [13]. The numerical value of $\boldsymbol{\mu}_{\mathrm{SI}}$ can only increase with the deepening of knowledge about the material world. The set of dimensionless criteria $\boldsymbol{\mu}_{\mathrm{SI}}$ does not exist in the physical reality. However, this observation is true for proper SI too. At the same time, the actually existing and observed object may be expressed by this set.

\subsection{SI and Class of Phenomena $(\mathrm{CoP})$}

3.3.1 SI is a set of the dimensional quantities, base and - calculated on their basis - derived, which are necessary and sufficient to describe the known laws of nature, as in the physical content and quantitatively [16].

3.3.2 SI includes a finite number of the dimensional base and derived quantities used for descriptions of different classes of phenomena $(C o P)$, which is depended on the chosen base quantities. In other words, the limits of the description of the studied material object are caused due to the choice of $C o P$ and the number of derived quantities taken into account in the mathematical model [16]. For example, in mechanics SI uses the basis $\{$ the length $\boldsymbol{L}$, weight $\boldsymbol{M}$, time $\boldsymbol{T}\}$, i.e. $C o P_{\mathrm{SI}} \equiv \boldsymbol{L M}$. 
3.3.3 $\boldsymbol{\beta}^{\prime}$ is the number of the base quantities of the chosen $C o P, \boldsymbol{z}^{\prime}$ is the total number of the dimensional quantities of the chosen $C o P, z^{\prime \prime}$ is a given number of the dimensional physical quantities recorded in the model, $\boldsymbol{\beta}^{\prime \prime}$ is the number of the base quantities recorded in the model.

\subsection{Uncertainty Relation of Modeling}

Equation (2) represents the conformity principle (uncertainty relation) for the model development process. Any change in the level of detail of the description of the observed object $\left(\boldsymbol{Z}^{\prime \prime}-\boldsymbol{\beta}^{\prime \prime} ; \boldsymbol{z}^{\prime}-\boldsymbol{\beta}^{\prime}\right)$ causes a change in the minimum comparative uncertainty $\boldsymbol{\Delta}_{\mathrm{pmm}} / \boldsymbol{S}$ of the model of a specific $C o P$ and the achieved accuracy of each main quantity characterizing the internal structure of the object. The apparent free choice of the range of changes $\boldsymbol{S}$ is limited by a fixed value of the comparative uncertainty $\boldsymbol{\varepsilon}$ for each $C o P$ chosen by the conscious observer. Expanding the range of changes $\boldsymbol{S}$ leads to a reduction of the achievable absolute uncertainty $\boldsymbol{\Delta}_{\mathrm{pmm}}$ and vice versa.

In other words: the conformity principle is a fundamental consideration, which establishes the accuracy limit (for a given class of phenomena) of simultaneously defining a pair of quantities observed by a conscious researcher, in particular, the absolute uncertainty in the measurement of the investigated quantity and the interval of its changes. It is important to realize that this conformity principle is not a shortcoming of the measurement equipment or engineering, but rather how our brains work.

It therefore turns out that the fuzziness (inaccurate representation) of the object in the eyes of the researcher depends both on the chosen class of phenomena and on the number of quantities taken into account by the conscious observer. The latter depends directly on the knowledge, accumulated life experience and intuition of the researcher. Objectively, these factors allow the possibility, already discussed above, of considering the choice of a quantity as a random process with an equally probable account of a particular quantity.

It is interesting to speculate on further applications of Equation (2) for the accuracy of the Planck constant measurement, which is discussed below.

\section{Applications of $\mu_{\mathrm{SI}}$ for Plank Constant Measurement}

Several scientific publications from 2007 to 2017 [18-27] were analysed from the position of the relative and comparative uncertainty values reached. The data are summarized in Table 1 . In order to apply a stated approach, as a possible changes interval of the Planck constant, we choose the difference in its value obtained from the experimental results of two projects: $h_{\min }=6.6260689124 \cdot 10^{-34} \mathrm{~m}{ }^{2} \cdot \mathrm{kg} / \mathrm{s}^{2}[18]$ and $h_{\max }=6.626071213 \cdot 10^{-{ }^{34}} \mathrm{~m}^{2} \cdot \mathrm{kg} / \mathrm{s}^{2}[19]$. Then the dimensional possible observed range $\boldsymbol{S}_{\mathrm{h}}$ of $h$ variations equals

$$
S_{h}=h_{\max }-h_{\min }=6.626071213 \cdot 10^{-34}-6.6260689124 \cdot 10^{-34}=2.3 \cdot 10^{-40}\left(\mathrm{~m}^{2} \cdot \mathrm{kg} / \mathrm{s}^{2}\right)
$$

Table 1. Planck constant determinations and relative and comparative uncertainties achieved.

\begin{tabular}{lllllll}
\hline \multirow{2}{*}{ Year } & $\begin{array}{l}\text { Plank } \\
\text { constant } \\
h \cdot 10^{34}\end{array}$ & $\begin{array}{l}\text { Achieved relative } \\
\text { uncertainty } \\
\mathrm{r}_{\mathrm{h}} \cdot 10^{8}\end{array}$ & $\begin{array}{l}\text { Absolute } \\
\text { uncertainty } \\
\boldsymbol{\Delta}_{\mathrm{h}} \cdot 10^{42}\end{array}$ & $\begin{array}{l}\text { h changes } \\
\text { range } \\
\boldsymbol{S}_{\mathrm{h}} \cdot 10^{40}\end{array}$ & $\begin{array}{l}\text { Comparative } \\
\text { uncertainty } \\
\boldsymbol{\Delta}_{\mathrm{h}} / \boldsymbol{S}_{\mathrm{h}}\end{array}$ & References \\
\hline 2007 & 6.6260689124 & 3.6 & 23.85 & & 0.1037 & {$[18]$} \\
2007 & 6.626071213 & 20 & 132.5 & & 0.5762 & {$[19]$} \\
2010 & 6.6260695729 & 4.4 & 29.2 & & 0.1268 & {$[20]$} \\
2011 & 6.62606912 & 29 & 192.2 & & 0.8355 & {$[21]$} \\
2011 & 6.626070082 & 3.0 & 19.88 & 2.3 & 0.8643 & {$[22]$} \\
2014 & 6.626069793 & 4.5 & 29.82 & & 0.1296 & {$[23]$} \\
2014 & 6.6260703412 & 1.44 & 9.54 & & 0.0415 & {$[24]$} \\
2015 & 6.62607004081 & 1.2 & 7.95 & & 0.0346 & {$[25]$} \\
2016 & 6.62606983 & 3.4 & 22.5 & & 0.0980 & {$[26]$} \\
2017 & 6.62606993489 & 1.3 & 8.61 & & 0.0374 & {$[27]$} \\
2017 & 6.62607013360 & 0.91 & 6.03 & & 0.0262 & {$[28]$} \\
\hline
\end{tabular}


The choice of the author of $\left(h_{\max }-h_{\min }\right)$ seems subjective and arbitrary. However, as already noted in 3.4, the magnitude of the product of absolute uncertainty in the measurement of the investigated quantity and the interval of its changes is fixed for a specific CoP. Therefore, the choice of the interval of observation of the measured quantity will not affect the final calculation of the recommended relative uncertainty. That is why, it can be argued that an objective, mathematically sound statement is given. We present the analysis of the Plank's constant measurements to convince readers that this metric is universal and, surprisingly, the results are trustworthy and not accidental.

It is seen from the data given in Table 1 that the findings presented in [29] are only partially confirmed. The fact is that in these studies there has been a dramatic improvement in the accuracy of the measurement of the Planck constant during the last decade. This is authorized as true when based on calculation of the relative uncertainty. At the same time, there was no mention of the Planck constant's achievable true-target value. Perhaps, this situation has arisen as a result of unaccounted systematic errors in these experiments. At the same time, it must be mentioned that, most likely, the exactness of Planck's constant, like other fundamental physical constants, cannot be infinite. Therefore, the conclusion is that there is room for further development to resolve these differences and find new ideas for a watt balance system with a more universal application, since the next generation of watt balance experiments is expected to become kilogram realization standards. The historical record suggests that there is still a need for proof that the Planck constant results are finally reproducible at an acceptable uncertainty [29].

In order to judge the data by the comparative uncertainty according to the proposed informationoriented approach, we need to calculate the lowest comparative uncertainty $\varepsilon_{L M T I}$ for electro-mechanical processes $\left(C o P_{\mathrm{SI}} \equiv \boldsymbol{L M T I}\right)$, which are usually used for the Plank constant measurements. In this case, it is required to equate its partial derivative with respect to $\boldsymbol{Z}^{\prime}-\boldsymbol{\beta}^{\prime}$, to zero. We can obtain:

$$
\begin{gathered}
\left(\Delta_{p m m} / S\right)_{Z^{\prime}-\beta^{\prime}}^{\prime}=\left[\left(z^{\prime}-\beta^{\prime}\right) / \mu_{S I}+\left(z^{\prime \prime}-\beta^{\prime \prime}\right) /\left(z^{\prime}-\beta^{\prime}\right)\right]^{\prime}=\left[1 / \mu_{S I}-\left(z^{\prime \prime}-\beta^{\prime \prime}\right) /\left(z^{\prime}-\beta^{\prime}\right)^{2}\right], \\
{\left[1 / \mu_{S I}-\left(z^{\prime \prime}-\beta^{\prime \prime}\right) /\left(z^{\prime}-\beta^{\prime}\right)^{2}\right]=0,} \\
\left(z^{\prime}-\beta^{\prime}\right)^{2} / \mu_{S I}=\left(z^{\prime \prime}-\beta^{\prime \prime}\right) .
\end{gathered}
$$

Thus, taking into account $(7),(9)$ and $(13)$, we get

$$
\begin{gathered}
\left(z^{\prime}-\beta^{\prime}\right)=\left(e_{l} \cdot e_{m} \cdot e_{t} \cdot e_{i}-1\right) / 2-4=(7 \cdot 3 \cdot 9 \cdot 5-) / 2-4=468, \\
\left(z^{\prime \prime}-\beta^{\prime \prime}\right)=\left(z^{\prime}-\beta^{\prime}\right)^{2} / \mu_{S I}=468^{2} / 38,265=5.723873 .
\end{gathered}
$$

where "-1" corresponds to the case when all the base quantities exponents are zero in formula (1); dividing by 2 indicates that there are direct and inverse quantities, e.g., $\mathrm{L}^{1}$ is the length, $\mathrm{L}^{-1}$ is the run length, and 4 corresponds to the four base quantities $\boldsymbol{L}, \boldsymbol{M}, \boldsymbol{T}, \boldsymbol{I}$.

Then, one can calculate the minimum achievable comparative uncertainty $\varepsilon_{L M T I}$

$$
\varepsilon_{\text {LMTI }}=\left(\Delta_{\text {pmm }} / S\right)_{\text {LMTI }}=468 / 38,265+5.723873 / 468=0.0244 .
$$

In the next step, we can argue about the order of the desired value of the relative uncertainty of $\mathrm{COP}_{\mathrm{SI}}$ $\equiv \boldsymbol{L} \boldsymbol{M T I}$. For this purpose, we take into account the following data: $\left(\varepsilon_{\min }\right)_{L M T I}=0.0244(16)$ and $\boldsymbol{S}_{h}$ $=2.3 \cdot 10^{-40}\left(\mathrm{~m}^{2} \cdot \mathrm{kg} / \mathrm{s}^{2}\right)(10)$. Then, the lowest possible absolute uncertainty for $\mathrm{COP}_{\mathrm{SI}} \equiv \boldsymbol{L} \boldsymbol{M T}$ equals:

$$
\left(\Delta_{\min }\right)_{\text {LMTI }}=\left(\varepsilon_{\text {min }}\right)_{\text {LMTI }} \cdot S_{h}=0.0244 \cdot 2.3 \cdot 10^{-40}=5.61 \cdot 10^{-42} \text {. }
$$

In this case, the lowest achievable relative uncertainty $\left(\mathrm{r}_{\min }\right)_{L M T I}$ for $\mathrm{COP}_{\mathrm{SI}} \equiv \boldsymbol{L M T I}$ is as follows:

$$
\left(r_{\min }\right)_{\text {LMTI }}=\left(\Delta_{\min }\right)_{\text {LMTI }} /\left(\left(\mathrm{h}_{\max }+\mathrm{h}_{\min }\right) / 2\right)=0.85 \cdot 10^{-8} \text {. }
$$

This value is in excellent agreement with the recommendations mentioned in [28], 0.91 $10^{-8}$, and should be satisfactory to the existing mass standards community. In this way, the ability to predict the Planck constant value by using the comparative uncertainty allows us to improve our fundamental comprehension of the complex phenomenon as well as to apply this comprehension to the solution of specific problems.

It is obvious that such findings will cause a negative reaction on the part of the scientific community. At the same time, an additional view of the existing problem will, most likely, help to understand the existing situation and identify concrete ways for its solution. The development of a larger number of 
designs and improvement of the various experimental facilities for the measurement of Planck's constant is an absolute must [30]. Reducing the value of the comparative uncertainty of the Planck constant obtained from different experiments, to the lowest achievable value of 0.0244 , will serve as a convincing argument for the professionals involved in the evolution of the SI.

It must be mentioned that CODATA has developed a method of doing the average, which is complex. In the frame of the CODATA technique to determine the recommended value of the relative uncertainty of this or that fundamental physical constant, a detailed discussion of the input data and the justification and construction of tables of values sufficient for the direct use of the relative uncertainty are conducted using modern advanced statistical methods and powerful computers. This, in turn, allows one to check the self-consistency of the input data and the output set of values. However, at every stage of data processing, an expert conclusion is also used, which is based on intuition, accumulated knowledge and the cumulative life experiences of scientists (one's personal philosophical leanings [31]).

The principal difference of the information-oriented approach in comparison with the "statisticsexpert" CODATA technique is the following. Within the framework of the presented approach, a theoretical and informational grounding and justification are carried out for calculating the relative uncertainty. A detailed description of the data and the processing procedures does not require considerable time, high quality staff and a significant budget.

\section{Conclusions}

It is obvious that reconciling the probabilistic subatomic world with the macroscopic everyday world is one of the great unsolved problems in physics. The use of the $\boldsymbol{\mu}_{\mathrm{SI}}$-hypothesis opens the opportunity to combine these two worlds.

The necessary and sufficient condition for choosing the right number of quantities recorded in the mathematical model describing the Planck constant measurement process is introduced.

The estimate of the a-priori achievable comparative uncertainty of the mathematical model caused by the limited number of the chosen quantities can be a peculiar metric for the adequacy of the accuracy of the physical experiments for the Planck constant measurements.

The method offers a tantalizing hint for more reliable predictability of the Planck constant and its relative uncertainty values than we might otherwise have anticipated by using the relative uncertainty calculated according to the "statistics-expert" CODATA technique.

The consequences of our conclusions are transferred to all models in physics and engineering, in which the above limit, due to a finite number of the quantities considered, is hidden, including measurement of fundamental physical constants, heat- and mass-transfer, thermal energy storage systems, and global climate models [13], where a compromise between complexity and achievable accuracy of model is always required. At the same time, $\mu_{S I}$-hypothesis is not inherent in nature; in other words, it is not a fundamental principle of nature. Indeed, it is not at all obvious that we can describe physical phenomena with the help of a single picture or mental representation. We form our models and representations by drawing inspiration from everyday experience. From this, we extract certain concepts, and starting from these, we invent, by simplification and abstraction, some simple models and clear concepts, which we then try to use to explain phenomena.

\section{References}

1. M. Henrion and B. Fischhoff, "Assessing uncertainty in physical constants," Amer. J. Phys., vol. 54, no. 9, pp. 791-798, 1986. http://goo.gl/wYwlBu

2. R. T. Birge, "The calculation of errors by the method of least squares," Phys. Rev., vol. 40, pp. 207-227, 1932.

3. M. G. Morgan, "Uncertainty: An introduction," CRAG Symposium, Uncertainty - from insight to action, pp. 162, 2013.http://goo.gl/LhyzVe

4. S. G. Rabinovich, Evaluating Measurement Accuracy- A Practical Approach. New York: Springer Science+Business Media, 2013. https://goo.gl/OEJYmY

5. R. Steiner, "History and progress on accurate measurements of the Planck constant," Rep. Prog. Phys., vol. 76, no. 1,pp. 1-46, 2013. http://goo.gl/s1GomR 
6. B. N. Taylor and T. J. Witt, "New international electrical reference standards based on the Josephson and Quantum Hall effects," Metrologia, vol. 26, no. 1, pp. 47-62, 1989.

7. I. M. Mils, P. J. Mohr, T. J. Quinn, B. N. Taylor and E. R. Williams, "Adapting the International System of Units to the twenty-first century," Phil. Trans. R. Soc. A, vol. 369, pp. 3907-3924, 2011.

8. A. D. Franklin, "Millikan's Published and Unpublished Data on Oil Drops," Historical Studies of Physical Sciences, vol.11, no. 2, pp. 185-201, 1981.

9. H. Arakelian, LMP Fundamental theory. Erevan: Armenian National Academy of Sciences, Sarvard Hrat Ltd., 2010.http://314159.ru/arakelian/arakelian1.pdf

10. NIST Special Publication 330 (SP330), The International System of Units (SI) 2008. http://physics.nist.gov/Pubs/SP330/sp330.pdf

11. S. L. Vasilenko, "Mathematics of Golden cross-section through the eyes of a philosopher," pp. 1-9, 2012, in Russian. http://goo.gl/mMerB3

12. A. S. Burrows and J. P. Ostriker, "Astronomical reach of fundamental physics," Proc. Natl. Acad. Sci. USA, vol. 111, no. 7, pp. 2409-2416, 2014. http://www.ncbi.nlm.nih.gov/pmc/articles/PMC3932888/

13. B. M. Menin, "Information Measure Approach for Calculating Model Uncertainty of Physical Phenomena," Amer.J. Comput. Appl. Math., vol. 7, no. 1, pp. 11-24, 2017. https://goo.gl/m3ukQi

14. V. N. Matveev and O. V. Matvejev, "Uncertainty relations as a consequence of the Lorentz transformations,"ResearchGate, pp. 1- 12, 2015. https://goo.gl/3gMRVA

15. L. Brillouin, Science and Information Theory. New York: Dover, 2004.

16. A. A. Sonin, The Physical Basis of Dimensional Analysis. 2nd edition, Department of Mechanical Engineering, MIT, 2001. http://web.mit.edu/2.25/www/pdf/DA_unified.pdf

17. L. Yarin, The Pi-Theorem, Experimental Fluid Mechanics. Berlin: Springer-Verlag, 2012. https://goo.gl/dtNq3D

18. R. L. Steiner, E. R. Williams, R. Liu and D. B. Newell, "Uncertainty improvements of the NIST electronic kilogram," IEEE Trans. Instrum. Meas., vol. 56, no. 2, pp. 592-596, 2007.

19. I. A. Robinson and B. P. Kibble, "An initial measurement of Planck's constant using the NPL Mark II watt balance," Metrologia, vol. 44, pp. 427-440, 2007.

20. P. J. Mohr, B. N. Taylor and D. B. Newell, 2012 "CODATA Recommended Values of the Fundamental Physical Constants: 2010," NIST 20899-8420.

21. A. Eichenberger, H. Baumann, B. Jeanneret, B. Jeckelmann, P. Richard and W. Beer, "Determination of the Planck constant with the METAS watt balance," Metrologia, vol. 48, pp. 133-141, 2011.

22. B. Andreas et al., "Determination of the Avogadro constant by counting the atoms in a 28Si crystal," Phys. Rev. Lett., 106, 030801, pp. 1-4, 2011.

23. S. Schlamminger, D. Haddad, F. Seifert, L. S. Chao, D. B. Newell, R. Liu, R. L. Steiner and J. R. Pratt,

"Determination of the Planck constant using a watt balance with a superconducting magnet system at the National Institute of Standards and Technology," Metrologia, vol. 51, no.15, pp. 1-22, 2014. http://goo.gl/hxLYTJ

24. C. A. Sanchez, B. M. Wood, R. G. Green, J. O. Liard and D. Inglis, "A measurement of Planck's constant using the NRC watt balance," Metrologia, vol. 51, no. 2, pp. 5-14, 2014.

25. CODATA recommended values of the fundamental physical constants: 2015 . https://goo.gl/zqzsrA

26. NIST-4 watt balance weighs in on Planck's constant 2016. https://goo.gl/jrM9tT

27. D. Haddad, F. Seifert, L. S. Chao, A. Possolo, D. B. Newell, J. R. Pratt, C. J. Williams and S. Schlamminger, "Measurement of the Planck constant at the National Institute of Standards and Technology from 2015 to 2016,"Metrologia, vol. 54, pp. 633-641, 2017.

28. B. M. Wood, C. A. Sanchez, R. G. Green and J. O. Liard, "A summary of the Plank constant determinations using the NRC Kibble balance," Metrologia, vol.54, pp. 399-409, 2017. http://sci-hub.tw/10.1088/16817575 /aa70bf

29. R. Steiner, "History and progress on accurate measurements of the Planck constant," Rep. Prog. Phys., vol. 76, 016101.pp. 1-47, 2013. https://sci-hub.tw/10.1088/0034-4885/76/1/016101\#

30. A. Eichenberger, G. Genev and P. Gournay, "Determination of the Planck constant by means of a watt balance," Eur.Phys. J. Special Topics, vol. 172, pp. 363-383, 2009.

31. D. Dodson, "Quantum Physics and the Nature of Reality (QPNR) survey: 2011," 2013. https://goo.gl/z6HCRQ 